\title{
Crianças de revistas (1930/1950)
}

OlgaBrites

PontifíciaUniversidadeCatólicadeSãoPaulo

\section{Resumo}

Asrevistasbrasileiras VidaDoméstica e Fon-Fon! publicaram en tre 1930 e 1959 di fe ren tes ma te ri a is so bre in fân cia: tex tos, fotografias, caricaturas, desenhos ilustrativos etc. Este artigo ana lisa al gu masima gens fo to grá fi cas ali pre sen tes, dis cu tin do seus cam pos te má ti cos e al guns pro ce di men tos de aná li se que con tribuíram para a ca rac te ri za ção das cri an ças no univer so so cial brasileiropelos periódicosindicados.

As cri an ças ne les re pre sen ta das eram, na sua ma i o ria, bran case pos su íam vida fa mi li ar es tru tu ra da (pai, mãe) e con di ção so cial privi le gi a da. As fo tos das cri an ças, em al gu mas cir cuns tân cias, eram pro du zi das em es tú di os fo to grá fi cos e des ta ca vam a beleza e felicidade. Seus trajes e adereços ajudavam a compor a ima gem de cri an ça bem nas ci da e fe liz.

0 universo da fotografia na infância está articulado a outras preocupações constantes quando se fala de criança: saúde, educação, religiosidade, lazer e moda. As fotos institucionais como, por exem plo, dos par ques in fan tis as su mem ca rac te rís ticas di feren tes da que las ex pli cita das an teri or men te. Nessecaso, as crian ças eram fo to gra fa das cole tiva men te como pro pa gan da dasativi da des desenvolvi das pelasins ti tui ções.

Considerando a fotografia como produção social, e evitando, por tan to, con ce bê-la como re flexo do real, o ar ti go pro cu ra articular textos e imagens, bem como refletir sobre diferentes possibilidades do mundoinfantil.

\section{Palavras-chave}

Infância - Imprensa - Fotografia. 


\section{Magazine children (1930/1950)}

Olga Brites

PontifíciaUniversidadeCatólicadeSãoPaulo

\section{Abstract}

The Bra zi li an ma ga zi nes Do mes tic Life and Fon-Fon! pu blished between 1930 e 1950 different ma terials aboutchild hood: texts, pic tu res, ca rica tures, illus tra tivedra wings etc. This article analyzes some of those photographic images, discussing the ir the ma tic fi elds and some analy sis proce du res that contributed to the cha racterization of children within the Brazilian social universe by those periodicals.

The children represented in them were, in their majority, white and they possessed structured family lives (father, mother) and a privileged social situation. The children's pictures, in some circumstances, were produced at photographic studios and they highlighted the beauty and happiness. Their clothes and accessories helped to compose the image of well-off and happy children.

The universe of photography in the childhood is articulated to other constant concerns when speaking about children: health, education, religiosity, leisure and fashion. Pictures of institutions such as, for instance, playgrounds assume characteristics different from those described previously. In that case, the children were photographed collectively as an advertisement of the activities developed by the institutions. Con sidering the pic tu re as a so cial production, and avo iding, the re fo re, to con ce $i v e$ it as a reflex of the real, the ar ti cle tries to ar ti cu la te texts and ima ges, as well as to con sider diffe rent possibilities of the child's world.

\section{Keywords}

Childhood - Press - Photography. 
Este artigo discute imagens de infância veiculadas em duas revistas brasileiras - Vida Doméstica e Fon-Fon! - no pe río do de 1930 a 1950.

Há um intenso investimento na infância du ran te esse pe río do, que se tra du ziu em ações pú bli cas, como a cri a ção do De par ta men to $\mathrm{Na}$ cional da Criança (DNCr, nos anos 40) e dos parques in fan tis na ci da de de São Pa u lo (na década anterior), dentre outras iniciativas.

Além disso, a imprensa periódica teve gran de cres ci men to a par tir do iní cio do sé cu lo XX (Sodré, 1977), assumindo os papéis de formadora de opinião pública, espaço de debate e produção ideológica, por meio da valoriza ção dasações governa men ta is dirigi das à infância, dentre outros tópicos. Colocou-se ainda como educadora da família - em especial, da mulher - no que se refere aos cu i da dos com a crian ça.

A periodização, entretanto, não represen ta uma ca mi sa de for ça, per mi tin do re fle tir so bre mo men tos an te ri o res, que ofe re cem condições de perceber permanências e mudanças em re la ção às ima gens de in fân cia cons tru í das por es saspublica ções.

As fo to gra fias de crian ças publica das por aque las revis tas fo ram con si de ra das como referen ci a is im por tan tes na cons tru ção de uma infân cia ide a li za da: ro bus ta, bela e ale gre. Esses atributos, explicitados em textos e imagens, são re corren tes para proje tar o fu tu ro de seja do: su ces so por meio de de ter mina da vida pro fis si onal ativa e es cola ri da de, cu i da do com a fa mília bemestruturada, saúde, beleza.

Embo ra não se jam obje to cen tral de dis cus são nes te ar ti go, os par ques in fan tis de São Paulo foram destacados por essas revistas como lu ga res privi le gi a dos para a edu ca ção infantil. Elas incorporaramuma discussão sobre taisins ti tu ições, presen te em diferen tes fon tes documentais produzidas pelo poder público, que des ta ca va sua fun ção de re ti rar as cri an ças das ruas e prepará-las para a vida social, dividindo com os pais a tarefa de educá-las convenientemente e participando da formação da própria família. ${ }^{1}$

Asima gens fo to grá fi cas de in fân cia trabalhadas em periódicos brasileiros dos anos 30 a 50 do século XX estavam em sintonia com ques tões como fra gi li da de, ame a ça so frida, ino cên cia, pers pec tiva de fu tu ro, pos sibili da des de trans for ma ção no pre sen te e ris cos de mor ta li da de. Daí, a ne ces si da de de cul tivar na que las pá gi nas uma vi são ide a li za da da criança, padrão a ser alcançado, como forte, saudável, estudiosa, adaptada ao ambiente familiar, escolarizada, religiosa, regrada, bem-comportada, com aspecto higienizado, li vre dos es tig mas vi síve is da ca rên cia. A po bre za in fan til, tam bém pre sen te nes sas pu blicações, figurava como problema, atraso e obje to de trans for ma ção para o bem do país.

A criança abordada nos periódicosera, muitas vezes, o bebê, que merecia cuidados médicos específicos (daí, seções dedicadas à pu e ri cul tu ra), e tam bém as mais cres ci das, em idade escolar.

\section{As revistas}

A pro du ção de ima gens de in fân cia obedecia a um ritmo específico de elaboração e difusão na imprensa periódica. Tal ritmo in cluía a retomada ou a criação de conteúdos com certaregularidade junto ao públicoleitor, com o surgimento de novas edições. Aquela peri o dicida de dizia respei to àveiculação de reportagens, fotografias, ilustrações, caricaturas e outrosmateriaissimilares.

0 pe ri o dis mo ti nha pa pel ativo no conjun to da vida so ci al, não po den do ser en ten dido como mero reflexo da sociedade. Assumia uma capacidade de interferir nos processos sociais gerais, estabelecendo arti-

1. Essas questões são discutidas no capítulo "Imagens de crianças: algumas experiências", da tese de doutoramento pela PUC/SP, intitulada Imagens da infância - São Paulo e Rio de Janeiro, 1930 a 1950, de nossa autoria. 
cu la ções en tre o uni ver so do con su mo e ou tras prá ti cas, crian do ne ces si da des, e con servan do há bi tos. As re vis tas e os jor na is não se li mi ta vam a registrar imagens de infância preexistentes no social. Eles participavam do fazer social daquelas ima gens, ope ran do nos pa râ me tros de um gru po so ci al e re for çan do seus valores.

Nas revistas como Vida Doméstica e Fon-Fon!, edi ta das no Dis tri to Fe de ral (Rio de Janeiro), fa la va-se para mu lhe res que pos su íam poder aquisitivo alto ou médio, abordando a in fân cia de seu meio so ci al, e ape nas even tu almente men ci o nan do o univer so da po breza.

Vida Doméstica e Fon-Fon! utilizavam diferentesrecursos de lin gua gem (tex to, fo tografia, desenhos), possibilitando o trabalho com a construção de sentidos no social por meio deelemen tos diversifica dos deexpressão.

Operiódico VidaDoméstica tra zia, além de ma te ri a is de pro pa gan da, tex tose ima gens a respei to de múl tiplostemasreferen tesà fa mília brasileira, como reportagens sobre cuidados com infância, casamento e moda, além de grande quantidade de fotos, cujos conteúdos re pre sen ta vam aquilo que o men sá rio con si derava even tosespecialmentesignifica tivos.

Arevis ta apre sen ta va-se como a pu blicação do lar e das mu lhe res e era pro pri e da de da Sociedade Gráfica Vida Doméstica Ltda., que possuía ou traspublicações: Vida Infantil, Vida Juvenil e seus res pec tivos al ma na ques.

Porsuasca racterísticas, VidaDoméstica era dirigida pri oritaria men tea o públi co feminino, e con ce bia a mu lher como con su mi do ra dos diversos produtos anunciados. Destacava aspec tos re fe ren tes à be le za (daí, anún ci os devári os cos mé ti cos: es mal tes, sa bo ne tes e cre mes), além de propagandas de tecidos para confecção de vestidos e outros produtos. As propagandas da revista abordavam os mais diversificados artigos e serviços:jóias, óculos, ca mi sas, carri nhos de be bês, re ló gi os, ci gar ros, móveis, lenços, porcelanas, brinquedos, remédios, alimentos, hotéis, agências de turismo, itens de moda. Ela dedicavagrande parte de suas páginas à publicidade, com muitos nú me ros que apre sen ta vam qua tro ou cin co pági nas ini ci a is ape nas de anún ci os. lsto serviu também para financiar o mensário, que teve um lon go pe río do de edi ção, de 1920 à pri me i ra me ta de da dé ca da de 60.

As pro pa gan das de VidaDoméstica reservadas à infância reforçavam as idéias de saúde, beleza e inteligência, apontando pro dutos que, milagrosamente, resolveriam todos os problemas: Toddy, Emulsão de Scott, Aveia Quaker etc.

Tal revis ta divul ga va tam bém anún cios dos perió di cos li ga dos a o seu gru po edi to rial, atra in do os le i to res para o que es sas publicações ofere ciam: calen dá rio, traba lhosma nuais, publicação de romance com ilustrações coloridas. Destacava-se ainda o luxo gráfico das edições, "re u nin do no mes da li te ra tu ra e das artesnacionais".

Esses anúncios realçavam a qualidade dos vários produtos lançados pelo mesmo gru po, que tam bém se des ti na vam a cri an ças e adolescentes.

Em 1940, Vida Doméstica trouxe repor ta gens que anun ci a vam seus 20 anos, re editando matéria do primeiro número, onde estampara seu projeto. Ali, o mensário afir mava "que não era novidade a existência de uma nova re vis ta no Bra sil ", su ge rin do o apare ci men to de vá ri as ou tras (So dré, 1977; Ba hia, 1990). Nesse quadro, no entanto, Vida Doméstica declarava destacar-se como diferente por tratardeassuntos úteis, como avi cultura e criação de outros animais (cães, ga tos), servin do ao en ri que ci men to do Bra sil, uma vez que dava aten ção es pe ci al ao de senvolvimento de suas terras. Propunha-se, as sim, a divulgaracon te cimen tos rela ci o na dosà agricultura e à pecuária, conclamando ao aprovei ta men to dasriquezasna tu ra is do país.

Tais considerações permitem observar que a própria vida familiar tinha um caráter urbano específico quando a revista surgiu, 
com um pú bli co mo ra dor de re si dên ci as onde ha via es pa ço para a cri a ção de ani ma is, a que o mensário tanto fazia referências, alcançando também, possivelmente, leitores de áreas rurais. Na con tra ca pa da revis ta, publi ca va-se freqüentementecalendário agrícola, o que se associava ao peso do público ru ral como seu alvo. Isso não impediu que a revista, referindo-se à in fân cia, de mons tras se pre o cupa ções com a ca rên cia de es pa ços ur ba nos de lazer, apontando apartamentos como novas moradias que dificultavam a brincadeira das crianças.

Também Fon-Fon! publicava materiais publicitários, em bo ra em me nor es ca la do que VidaDo més tica, e apre sen ta va pro du tos e serviçosse melhan tesàqueles men ci o na dos. Iniciando-se em 1907 (Silva, 1990), Fon-Fon! definia-se como "semanário alegre, político, crítico ees fuzian te", explo ran do larga men teo desenhohumorístico, semsedirigirprio ritariamente ao público feminino, mas trazendo em suas crônicas e outros textos ou imagens alguns ape los para aque le univer so de con su motemas como a flor e o sorriso, for mas de conquis tar um ma ri do, ali men ta ção ade qua da para a nutriz etc.

Essas duas revistas diferenciavam-se na me di da em que VidaDomés tica era mu i to mais di ri gi da à fa mília e a o lar, en quan to a ou tra publi ca ção se de di ca va pri o ri ta ri a men teà ca ricatura e à política, realçando o espaço carioca. Bassanezi (1996) salientou a preponderância da crô ni ca so ci al em suas pá gi nas. Essa ten dência diminuiu sensivelmente nos anos 30/40, quando Fon-Fon! pu bli cou fo to gra fi as de mulheres como modelos de elegância e beleza, jun to a ima gens de pes so as de am bos os se xos, formadas em diferentes cursos universitários, embora as figuras femininasfossem mais des ta ca das nos uni ver sos de luxo e be le za que nos da educação.

Fon-Fon! explorava as situações de hu mor, nas quais era possível rir de temas que, noutras circunstâncias, se revelavam muito mais dra má ti cos - al co o lis mo, por exem plo. 0 riso também assumiu ocasionalmente a função de repor papéis tradicionais para a mulher, conservan do normas. ${ }^{2}$ A própria me di ci na serviu de obje to para o riso, re for çan do, por vezes, a imagem de ignorância do paciente, mas também atribuindoao médico uma po ten ci al ame a ça à vida do do en te. ${ }^{3}$

Nesse contexto, as crianças, principalmen te os me ni nos, apa re ciam comoleva dase desafiadoras do mundo adulto. ${ }^{4} \mathrm{~A}$ narrativa qua driniza da "Crian ças deHoje" mos trou pais ar ru man do presen tes em árvo re de Na tal para a filha, que os ob serva va sor ra te i ra men te; em se gui da, quan do eles se re co lhe ram, ela foi à árvore e mexeu nos brinquedos deixados pelos pais; por fim, es tes fo ram acor dar a fi lha para lhe dar os pre sen tes e ela se com por tou como se nada houvesse acontecido (Fon-Fon!, jan. 1949).

Esse último material apresentou uma cri an ça es perta, com va lo res ex pres sos no seu tem po pre sen te, que do mi na va o mito de $\mathrm{Pa}$ pai Noel sem re nun ci ar às suas van ta gens. 0 riso, nos exem plos ar ro la dos, não se com pro metia rigidamente com nenhum ponto de vista, fun ci o nan do como ins tru men to de acolhi men to para de ter mi na das ques tões e de rejeição para outras (Silva, 1989). 0 que in te res sa em re la ção ao riso é pen sar so bre arti cu la ções en tre os te mas abor da dos e ou tros materiais dos periódicos.

2 Uma mulher pergunta ao marido: "Querido, por que não fazes um seguro de vida?" Ele responde-lhe: "Por que? Vais principiar de novo a cozinhar?" (Fon-Fon !, maio 1942).

3. Um médico indagou ao paciente: "Fizeram-lhe bem as sanguessugas que lhe receitei ontem?". 0 doente respondeu: "Não sei, doutor, a única coisa que posso adiantar é que tinham um sabor detestável". Noutro exemplo, uma paciente declarou: "Sofro muito, Doutor. Algumas vezes, tenho ímpeto de matar-me". 0 médico rebateu: "Não se aflija tanto, Senhora, para que eu estou aqui?". Noutra ocasião, alguém perguntou a uma mulher: "E seu marido, já está fora de perigo?". A resposta feminina foi: "Ainda não, pois o médico continua indo lá em casa todos os dias" (Fon-Fon !, jul. 1942).

4. Num desenho, apareceram homem, elefante e dois moleques correndo, com o primeiro declarando: "Seus moleques! Eu os ensinarei a por sabão na água do elefante!” ( Fon-Fon!, jan. 1938). 
Predominavam propagandas e fotos nas páginas de Vida Doméstica. Como em Fon-Fon!, os tex tos que acom pa nha vam as fotografias eram curtos, apenas explicavam os eventos, quase sempre marcados por luxo e grandiosidade, com homens e mulheres vestidos em es tiloso fis tica do. Havia, portan to, um modo de vida que era afirmado e reforçado para as le i to ras como ide al, pró xi mo das ca madas mais privile gi a das so cial men te, que apa reci am como exem plos de ele gân cia e be le za. As ima gens da in fân cia es ta vam sin to ni za das com esse pa drão adul to de viver o so ci al, e atri bu tos dos gru pos so ci a is privi le gi a dos tam bém eram de sig na dos para o univer so in fan til.

Repetiam-senelas, todavia,instrumentos para atingir seus objetivos gerais de vigiar mães, in fân cia e fa mília: va lo ri za ção da pu e ri cul tu ra e a ne ces si da de de con tro lar o tem po da criança.

\section{Crianças fotografadas}

As seções "Página Infantil” e "Crianças" de Fon-Fon! dedicavam-se a publicar retratos de crianças, tanto bebês como as maiores. Os

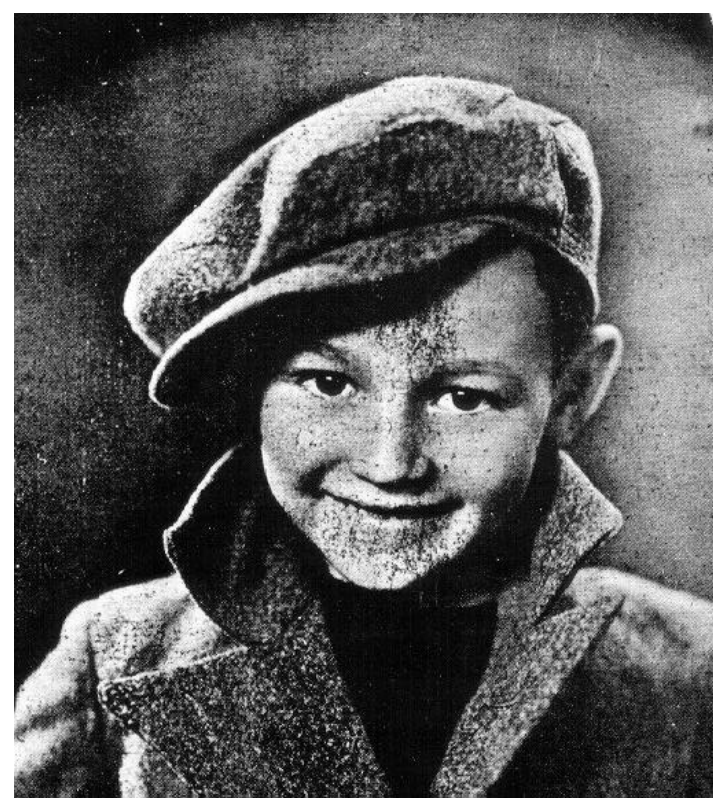

Infância, dez. 1935 meninos crescidoseram fo to grafados, habitualmente, com roupas mais sérias, como miniaturas de adultos - terno, dól mã. As me ninas figuravam em trajes mais propriamente infantis, embora, em alguns casos, a pose para a fotografia revelasse postura composta, na direção da seriedade. Podemos verificar diversasima gens de infân cia cons truídas pelas revistas

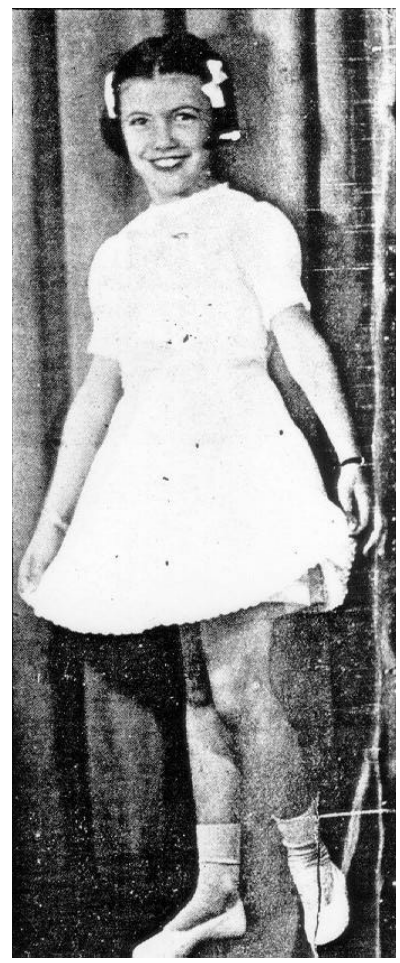

Fon Fon!, 22 jun. 1940 por meio de poses, roupas e adereços.

A fo to gra fia não é re fle xo do real nem ilus tra ção, ela é do ta da de uma his to ri ci da de pró pria, que con si de ra novas tec nolo gias, formas de con ce ber e en ca rar o so ci al. 0 fo tó grafo, por seu tur no, não é mero es pec ta dor do obje to fo to gra fa do, ele age ein ter fere, criando novasrea lida des.

Nessas fotografias de crianças, a pobreza não costumava aparecer, inexistindo espaço para o registro do trabalho infantil, da suje i ra, da ca rên cia, ex ce to sob o sig no da as sis tên cia. Aluz que revelava es sas cri an ças fa zia par te de um mun do da bela apa rên cia, que indicava saúde e felicidade. Tirar fotos de cor po in te i ro in te gra va um proje to de revelar condições sociais favoráveis: o corpo era ele men to im portan te no diag nós ti co dos fo to gra fa dos - pés, mãos, ros to, tron co, roupas asseadas, limpas, cabelos impecáveis, pele aveludada e macia, sem prebran ca, tão valorizada no anúncio de certos produtos para a in fân cia. A lin gua gem fo to grá fi ca era 
parte de um universo marcado por debates e trocas de experiências - iluminação, ambienta ção, a de reços de co ra tivos que fa cili ta vam as po ses (Ma cha do, 1984; Kos soy, 1989; San tos, 1989).

A pri me i ra co mu nhão das cri an ças - sa cra men to da lgreja Ca tó li ca - fez par te des ses eventos comemorados socialmente e mereceu re gis tros fo to grá fi cos nos dois pe rió di cos analisados. Do cu men tar a pri me i ra co mu nhão reme tia, ain da, a uma di men são de re li gi o si da de a ser pre ser va da. 0 tex to que acom pa nha uma das fo tos de cri an ças nes sas con dições apre senta va os se guin tes di ze res:

No dia em que Je sus vem ale grar o co ra ção dos pequeninos. É uma data inesquecível na vida das cri an ças, a da sua pri me i ra co mu nhão. Fica assinalada para o resto da existência, como a me mó ria fe liz da pu re za in te gral da me ni nice. A graciosa menina Noemia Machado no dia em que se aproxi mou da Mesa Eu ca rís tica e re a lizou a ma i or as pi ra ção de sua exis tên cia in fan til.É dileta filha do sr. Adolfo Machado. (Vida Doméstica, jan. 1930)
Nessa perspectiva, a felicida deabsoluta das pessoas (e, mais ain da, das cri an ças) residia na fé cristã, que devia se fazer pre sente em todos os lares. A imagem de ino cência da criança foi associada a essa dimen são de reli gi o si da de, a ser preserva da. Tal espiritualidade pressupunha identificar uma fili a ção, de mons tran do não se tra tar de uma cri an ça qual quer. Essa ati tu de não era exclusiva para a criança de classe média, como se observava em festas de Natal em instituições assistenciais, públicas e privadas, freqüentemente noticiadasem revistas e jor na is, em que eram fo to gra fa das per so na li da des e o públi co ali aten di do, de monstrando a união de todos em Cristo e até a iden ti fi ca ção en tre cri an ças po bres e Je sus. $\mathrm{Na}$ criança e pela criança, diluíam-se ten sões e diferenças sociais. As fotografias apresentam uma classe dominante sintoniza da com o univer so da po bre za, aju dan do, transformando, diferenciando-sedese to res de elite alheios a esses problemas. Tratava-se de exi bir os po bres e, jun to com eles, a elite que o país devia desejar.

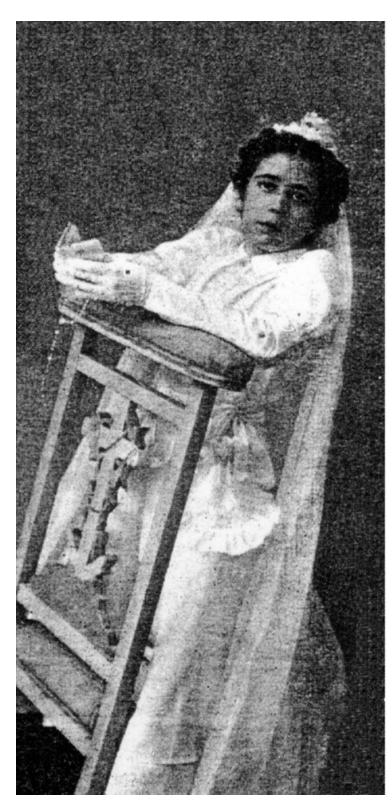

Fon Fon!, 21 out. 1939

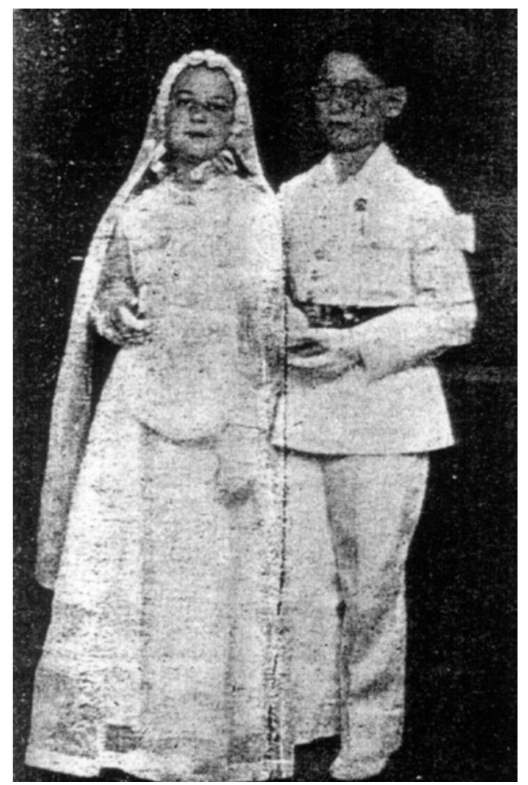

Fon Fon!, 25 nov. 1939

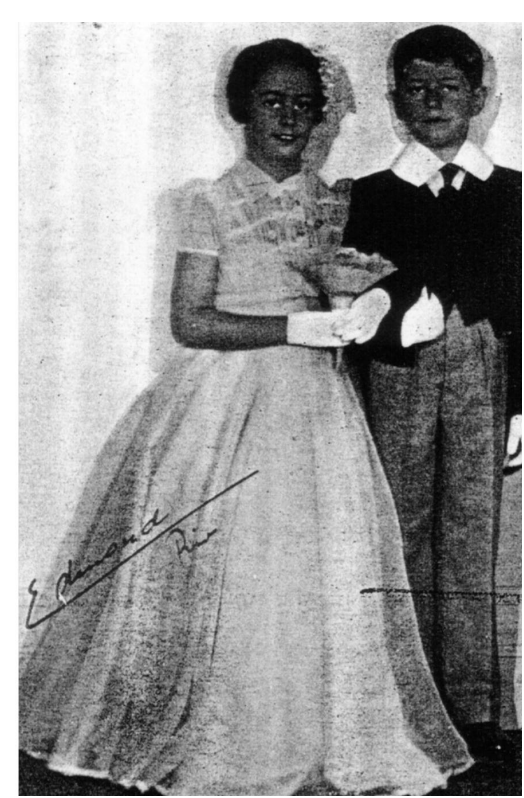

Fon Fon! , 23 dez. 1939 
Fo to gra fias de primeira co mu nhão in cluiam trajes pomposos, semelhantes a roupas nup ci a is - ves ti do lon go, ter no ou mes mo fraque-, re me ten do o mun do in fan til ao univer so adul to. Em vá ri as oca siões, cri an ças mais cresci das, de am bos os se xos, sur gi ram car re gan do ade re ços da vida es co lar - li vros, por exem plo. Animais de estimação e brinquedos também apa reciamjun to aosseus do nos, es pecialmen te os menores, em situações de pose calculada. Brin que dos ou ani ma is re me ti am ao con su mo, status, tudo mu i to dis ci pli na do, ex pon do uma imagem de infância num padrão social de privilégio.

Era mais ha bi tual fo to gra far cada per so nagemindividualmente, procedimento modifica do qua se ape nas na pri me i ra co mu nhão e no car na val. Embo ra os pais não fi gu ras sem jun to aos filhos, seus no mes eram sem pre as so cia dos aos das cri an ças, di vi din do com es tas o pres tí gio da exposição pública.

Essas fo tos va lo ri za vam a pose, su ge rin do situações de estúdio fotográfico, fixando um mo men to da vida des sas pes so as (Bart hes,

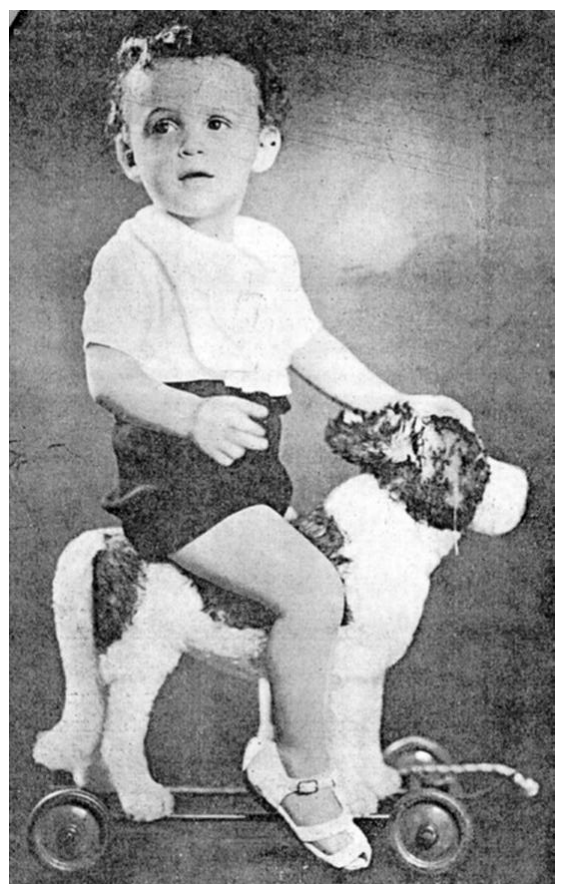

Infância, jul. 1936
1996), e cons ti tu in do uma re fe rên cia de prestí gio no meio fa mi li ar e so ci al. As fo to gra fi as de crianças na quelas se ções de Fon-Fon!diferi am de ima gens fo to grá fi cas usa das em propaganda, em que, por vezes, a exibição do pro du to nem sem pre exi gia que as fi gu ras huma nas olhas sem para o públi co le i tor. Evidenciavam, todavia, a importância da pose fotográfica, até em situações que aparentavam informalidade, como salientado por
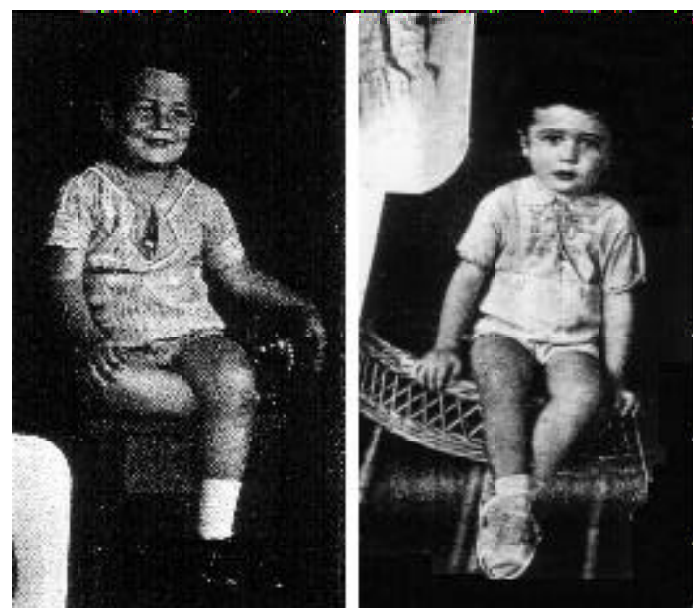

Fon Fon!, 03 set. 1938

Denise Bernuzzi Sant'Anna (1997), referindo-se a outrosmateria is do periodismo.

As experiências fotográficas de Vida Doméstica diziam respeito a certos ritos de passagemvalorizadossocialmente - primeira comunhão, casa men to, en cer ra men to de ano le tivo, for ma tu ras-, su ge rin do como eles deviam ser comemorados e rememorados: rou pas, cerimônias, exibição de zelo e cuidado. Por ou tro lado, os adul tos fo to gra fa dos re presen ta vam di men sões da vida "bem su ce di da", resul tan te de fa mílias es tru tura das. Taisfo togra fi as ex pres sa vam sin to nia com va lo res sociais dominantes.

A família do presidente Vargas tinha também destaque nesses ritos fotográficos, como mo de lo de fi lhos bem-criados e exemplo para o Bra sil (VidaDoméstica, jan. 1938), imagem pater nal e fa mi li ar que foi dis cu ti da, 
a par tir de ou tras fon tes, pela his to ri a do ra Maria Helena Capelatto (1996).

Páginas que noticiavam casamentos por meio de fotos eram freqüentes, com meninas que apa re ci am como da mas de hon ra. Fo to grafi as de no ivas pa u lis tas tam bém fo ram publicadas em vá ri os nú me ros da revis ta, des ta can do o luxo. Ao mes mo tem po, o ca sa men to privilegiado era aque le re a li za do na lgreja Ca tó lica, num contexto de pompa. Não havia espaço para o re gis tro de re la ções ex ter nas àque les pa drões concubinatos, mães solteiras, relações amorosas eventuais, roda dos expostos -, situações que Maria Odila Silva Dias (1984) indicou em relação ao século XIX e que continuavam a ocorrer no período abordado.

Existiam também fotografias de cerimôni as des sa na tu re za em âm bi tos po pu la res, fe itas por fo tó gra fos am bu lan tes, que não che ga vam àque le es pa ço de publi ca ção, evi den cian do a assimilação da linguagem fotográfica por es ses grupos sociais dentro dos seus padrões de vida (roupas, ambientes etc.), como consta de lem bran ça pes so al de fo to gra fi as de meus pais, bem como de de po i men tos de co nhe ci dos so bre fotografiaambulante nos anos 40 e 50 .

Retratos infantis, que realçavam a filiação das cri an ças e a in fân cia como ex pres são de bele za, ale gria e feli ci da de na fa mília, fo ram divulga dospor Fon-Fon! des de os anos 20, jun to com fo to gra fi as de ca sa men tos, mo ças, for maturas e bodas de prata (Fon-Fon!, jan./fev. 1926).

Em VidaDoméstica, as cri an ças apa re ciam fotografadas em várias solenidades, como audição em conservatório musical, o que não deixava de ser propaganda da própria escola, apontando uma musicalidade que educava de acor do com a psi co lo gia e a di dá ti ca.

Meninos e me ni nas fo ram tam bém fo togra fa dos em ba iles car na va les cos, com suas diver sas fan ta si as. A ima gem da in fân cia, nes ses ca sos, foi sem pre de su ces so, es pe tá culo, gra ça, beleza,felicidade.

Eram comuns as fotografias de crianças

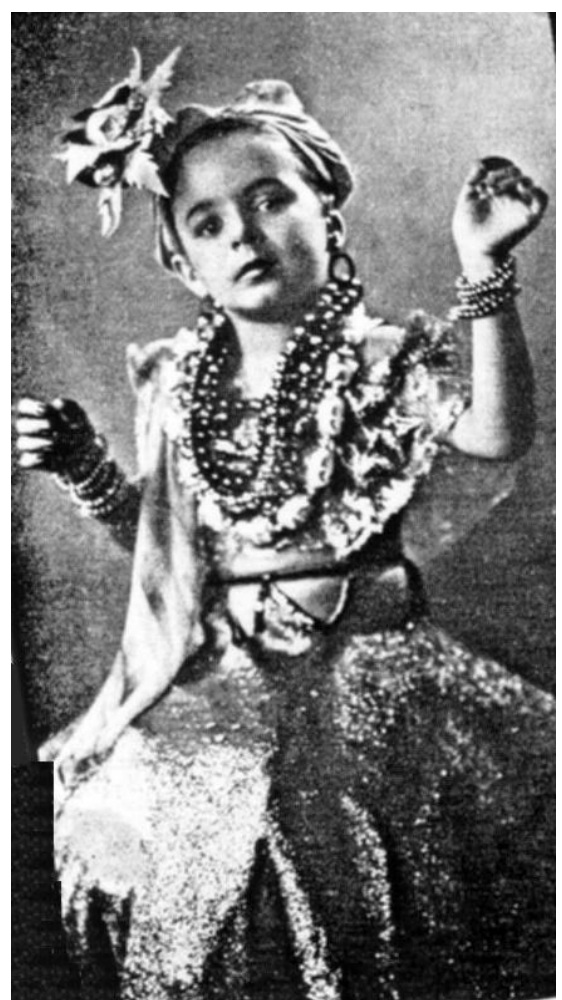

Fon Fon! , 22 jun. 1940

no en cer ra men to do ano le tivo. So bre um desses eventos, uma das revistas escreveu:

Estas fo to gra fias en can ta do ras são da fes ta de encerramento do curso primário do Colégio Jacobina. Encanto, graça, dois adjetivos que ca bem tão bem à in fân cia, ali se en con tra vam para alegria dos assistentes, pais e pessoas ami gas das fa mí li as das alu nas, que não se esqueceram de aplaudir as pequenas artistas. Momentos da infância que nunca mais serão es que ci dos, es tas fes tas de fim de ano têm o dom de deixar uma recordação agradável de um ano pas sa do no con ví vio es co lar, para que mais tar de pos sam sen tir como nos ver sos do po e ta, as sa u da des da in fân cia que ri da que os anos não trazem mais. (Fon-Fon!, jan. 1950)

A imagem idealizada da infância expressou-se em alusão a bons tempos. As re cordações desse período deveriam ser preenchidas pela alegria que representava 
vi vê-la, eta pa “ri so nha e fran ca”, que de via re tornar por meio de lembrançaspositivas.

Vida Doméstica identificou quais momentos da infância o leitor precisava guardar na lem bran ça, in cen tivan do o re gis tro fo to gráfico como instrumen to ade qua do para preservar recordações de festas e momentos de sucesso.

Esse mes mo tom foi usa do pelo pe rió di co no registro de diversos eventos, como as for maturas da Faculdade Nacional de Medicina, da Univer si da de do Bra sil, e dos Co lé gi os Companhia Santa Tereza de Jesus, Bennett, Nossa Senhora de Sion, Anglo-Americano e Notre Dame, abran gen do adul tos e se guin do os mesmos pa drões de luxo e so fis ti ca ção as si na la dos em re la ção ao mun do in fan til. To das es sas insti tu i ções de en si no eram de eli te, o que aju da a si tu ar o âm bi to de aten ção e de pos sível cir cula ção de Vida Doméstica.

Ou tra se ção des sa re vis ta que ori en ta va no cu i da do dos fi lhos, "Guia das Mães”, apre sen ta va-os como as "melhores boas festas de casais ven tu ro sos", e eram mu i tas as fo tos de cri an ças en vi a das para se rem pu bli ca das, com tex tos curtos, queiden ti fica vam os fo to gra fa dos - procedimento freqüente da imprensa brasileira até esse período,visando a angariar rendas (Sodré, 1977). 0 men sá rio co lo ca va os ser vi ços de fo tó grafos à disposição dos leitores, circunstância que re for ça in ter pre ta ções aqui fe i tas so bre a inten ci o na li da de e a his to ri ci da de do ato fo to gráfico. Eis dois exemplos daquelaslegendas:

Li a mir, com três anos e meio, fi lhi nho do sr. F. Guerriére e de sua exma. es posa, dona Ade lina Guerriére, cirurgiões dentistas. 0 interessante filhinho do Dr. Agostinho C. Brêtas, médico. (VidaDoméstica, jan. 1930)

Registrava-se sem prea prestigia da atividade profissional dos pais, indicando ligações da in fân cia des ta ca da com status so cial. Nes ses termos, tais fotografias funcionavam como uma espécie de publicidade pessoal e familiar, fortalecendo publicamente as imagens daque las pesso as. Arevis ta tam bém apresentava retratos de personalidades públicas, como o pre si den te Vargas. Divul gar fo to gra fi as e nomes de fa mí lia era um ges to de apro xi mar es ses personagens daquele universo de conhecimento públicoampliado.

Essas imagens de crianças não combina vam com os ín di ces alar man tes de mor ta lidade infantil, mais presentes na imprensa diária paulistana (O Estado de São Paulo e Diário Popular) e eventualmente referidos naquelas revistas. A insistência das últimas em publicarma téri as reserva dasà pu ericul tura ex pres sou pre o cu pa ção de pre servar a vida de crianças, inspirada no modelo do adulto bem-sucedido. Por ou tro lado, a cri an ça fo tografada naquelas circunstâncias ritualísticas aparecia integrada ao mundo adulto, demonstrando relação harmoniosa entre pais, filhos e fotógrafo, e evidenciando a circulação des sesva lo res en tre adul tos e cri an ças.

Esse universo apontado pelas revistas estava, em certos aspectos, diferenciado daqui lo que era pa râ me tro para as ca ma das po pulares. 0 uni formenas es colas des tina dasaos po bres, por exem plo, era uma for ma de evi tar a aparência indesejada das crianças, representando também, simbolicamente, igualda de de con di ções. Em es co las de eli te, ao me nos até os anos 50, uni for mes eram usa dos como sím bo lo de ostentação, incluindodivisão entre roupas para o co tidia no e aquelas des ti na das a des files cívicos e festas - tecidos ainda mais finos, acom pa nha dos de cha péus e lu vas etc.

Também para crianças trabalhadoras, comojornaleiroseven de do resambulantes, os uni for mes eram uma ma ne i ra de evi tar tra jes indesejados, maltrapilhos, identificando-as no es pa ço urba no, disciplinan do suasatividades, e separando trabalhadores infantis de menores delinqüentes, e ambos das crianças bem-nascidas.

As cri an ças fo to gra fa das em ativi da des escolares ou exibições cívicas não eram 

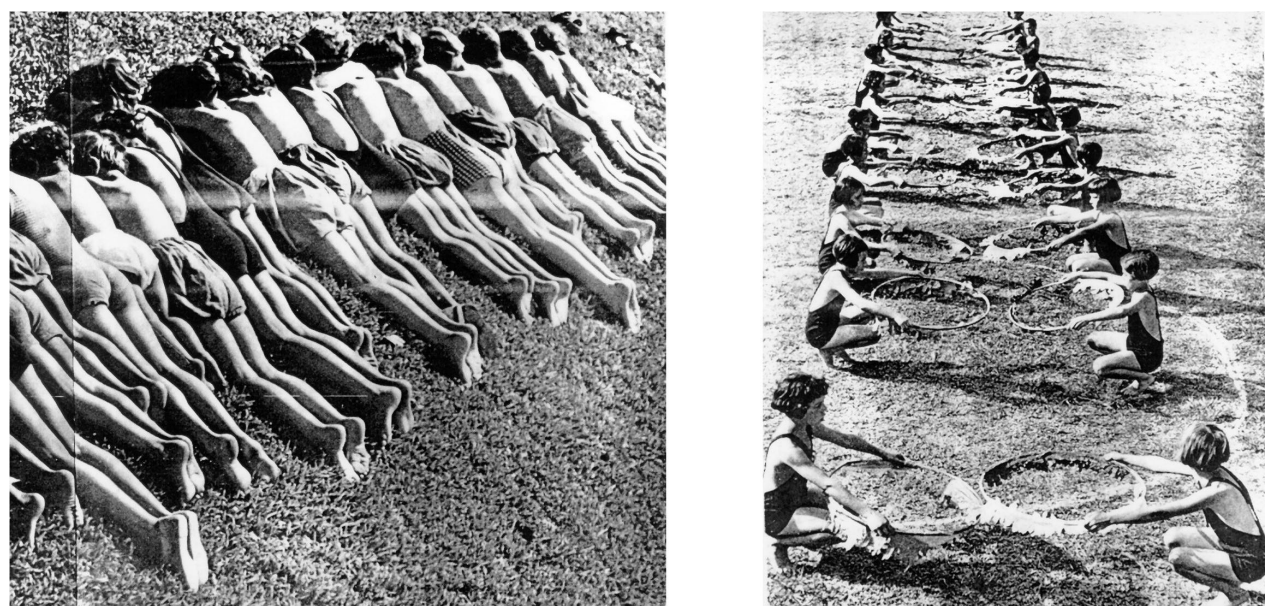

Par ques Infan tis-De par ta men to de Cul tu ra

apresentadas individualmente. As fotos conside ra vam sem pre gran de quan ti da de de alu nos, como nos par ques in fan tis, uni for miza dos, disciplinados, exercendo atividade física orientada, de mons tran do que a pre o cu pa ção prin cipal era valorizar iniciativasdas instituições conside ra das, le van do em con ta a idéia de que o projeto era bem recebido por um número expressivo de crian çase fa mílias.

Em al guns mo men tos, hou ve crí ti cas aos shorts usa dos por me ni nas e mo ci nhas em desfilesna cida de, julga dos "su má ri os", exibidores do físico. Aim pren sa, fa zen do es ses co men tá rios, assumia-os como pertinentes, apontando que eles sur gi ram em "ro das de pes so as res pe ita das". 0 tema apa re ceu nojor nal pa u lis ta no $A$ Gazeta (1937).

Fon-Fon! ofereceu sugestões de roupas para cri an ças, apre sen tan do mo de los e mol des, indicando tecidos finos: organdi, cambraia de li nho bran co etc. ( Fon-Fon!, jan. 1938). Cos turar era desaconselhadoapenas em alguns momentos da gravidez, levando em conta os cu i da dos para pre ser va ção da sa ú de da mãe e da criança (Fon-Fon!, fev. 1942).

Recomendava-se simplicidade nos trajes infantis, definindo o que se considerava bom-gosto e atitudes inadequadas nas vestimen tas. Tam bém aquijul ga va-se ne ces sá rio observar a criança, para definir momentos significa tivos de suavida. Evi den te men te, essa cri an ça ti nha uma iden ti da de so cial es pe cífica de clas se mé dia e a ati vi da de da cos tu ra também aparecia como tarefa doméstica da mu lhernessa faixa so cial (Fon-Fon!, jun. 1942).

Considerando que a linguagem fotográfica necessitava de um repertório de conhecimentos do leitor, sua decodificação requeria referenciais, demonstrando que tal leitura não dependia apenas de uma visão imediata. A seção "Teste Fotográfico", de Fon-Fon!, exi gia do le i tor que ele iden ti fi casse as pessoas fotografadas através de múltiplas op ções, à ma ne i ra de um tes te ( Fon-Fon! , out. 1949).

Jun to com a fé, a ima gem fo to grá fi ca da criança foi também associada ao poder de Estado, como se observa em cena de Getúlio Vargas com trigêmeos, vestidos como marinhe i ros, o pre si den te se gu ran do a mão de um de les. Se gun do a le gen da des sa ima gem, Vargas ama va as cri an ças tam bém como edu ca dor que era, fun ção que, nas pá gi nas da re vis ta, se deslocava entre médicos, mães, professores e ou tros ato res so ci a is. Os tri gê me os fo ram usadoscomosímbolo do pen sa men to presiden cial sobre o futuro, coadjuvandouma legitima ção dessa autoridade( Fon-Fon!, mar. 1942).

Heloísa Helena de Jesus Paulo (1987) co men ta a pro du ção de car ti lhas, santinhos e 
ou tros ma teria is des ti na dosaosjovens nesse período, conferindo-lhes "um papel de destaque na edi fi ca ção de uma na ci o na li da de". Capelatto (1996) realizou análise comparativa da propaganda varguis ta e pe ro nis ta, sa li en tan do o menor ape lo emo ci o nal nos exem plos bra si le iros.

Essas fotografias de crianças tiveram o pa pel de tor nar ain da mais pal pá ve is os te mas aborda dos (beleza, robustez, ale gria, ele gân cia, ri que za), ex plo ran do o po der de conven ci mento delegado à visão. A rapidez de articulação en tre as sun tos e per so na gens com base em foto gra fi as foi im por tan te di men são nasima gens de in fân cia des se pe río do, si tu an do-as em múlti plos con tex tos da vida so cial.

Em Fon-Fon! ocorreu procedimento similar, que pode ser exem plifica do coma fo tografia do Dr. Paulo Frontin, homem público, cuja con di ção de pai foi va lo ri za da por ad je ti va ção po si tiva, la de a do pe los filhos-duas meninas e um menino (Fon-Fon!, dez. 1907). Embo ra esse exem plo seja do iní cio do sé cu lo, a prática permaneceu na revista até o periodo aquidiscutido.

As le gen das de fo to gra fi as em Fon-Fon! identificavam meninas como "graciosas" ou "in te res san tes", en quan to os me ni nos eram designados como "inteligentes". Essa diferença in di ca que as ima gens de in fân cia tam bém participavam das identida des de gênero.

Schemmes (1995) destacou o papel das fes tas cívi cas na con so li da ção da idéia do novo e no apoio ao governo Vargas, afir man do que desempenhavamim portan tepa pel na "co op tação das mas sas" e no "dis ci pli na men to dos ci dadãos", "considerando-se fundamental a par ti ci pa ção das es co las, das cri an ças e das mulhe res, além dos ho mens da Esco la de Edu ca ção Físi ca do Exér ci to,Polícia, Ti ros de Gu er ra etc." (Schemmes, 1995).

Um lugar privilegiado da cidade de São $\mathrm{Pa}$ u lo para re gis trar a pre sen ça de cri an ças em si tu a ções so le nes, como o Dia da Cri an ça, foi o Parque da Água Branca. Fotos panorâmicas mos travam a gran de quan ti da de de crian ças em ativi da desfísicas. A prática da edu ca ção física era elo gi a da como be né fi ca a mu lhe res e crianças, sendo lembrados o Departamento de Educação Física do Estado de São Paulo e a Cru za da Pró-Infância. No caso do "Con cur so de Ro bus tez Infan til”, os be bês eram fo to grafados de fraldas, sentados, de peitos nus. 0 jornal destacou como símbolo dessa campanha uma cri an ça nua numa ba lan ça ( OEstado de São Pa u lo, nov. 1936). Tam bém fi gu ra ram crianças vestidas, apresentadas pelas mães sor ri den tes, que exi bi am os fi lhos para o pú blico leitor como frutos de grande cuidado, numa ati tu de de or gu lho.

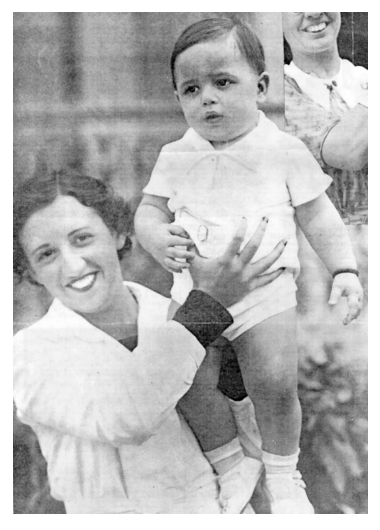

\section{Infância,} out. 1936

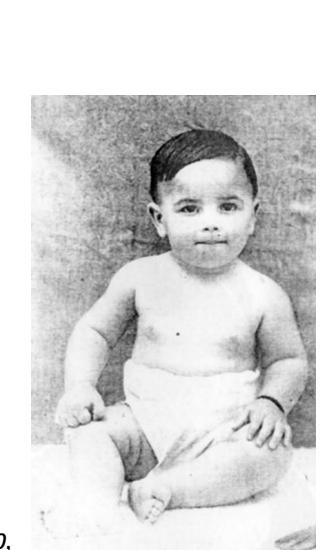

Re vis ta de São Pa u lo, out. 1936

\section{Política institucional e pobreza}

As ações governamentais mereceram sem pre elo gi os porparte de VidaDoméstica e outros órgãos congêneres. O Departamento Nacional de Propaganda foi ali apresentado como ór gão que atu a va de for ma in teligen te, por exemplo, na produção cinematográfica oficial, servindo de elemento para orientar ou tras re a li za ções nes sa área, no Bra sil ( Vida Doméstica, jan. 1939). A revista apresentava-se como de di ca da às re a li za ções do "Re gime Na ci o nal”. A partir dis so, po de mos afir mar 
que as imagens de infância divulgadas pelo mensário estavam em sintonia com projetos políticos mais amplos, desempenhando neles um pa pel ativo.

É de mons tra tiva dessa di men são a re porta gem “O Bra sil é uma De mo cra cia Edu ca ti va”, caracterizando a política interna brasileira como "edu ca do ra, de as sis tên cia, de dis ci plina e controle de todas as atividades individuais" (Vida Doméstica, mar. 1941) .

As no ções de tra ba lho e or dem fo ram empre ga das de for ma a qua li fi car as ações go vernamentais. 0 Estado acolheria a todos, sem dis tin ção de na ci o nali da de ou reli gião. 0 presiden te foi apre sen ta do como sím bo lo de uni dade da nação.

A democracia foi defendida e definida como o sis te ma po lí ti co que ti nha por pres supos tos a li ber da de e a igual da de, aque le que vinha do povo e se voltava para ele, uma forma de or ga ni za ção que se opu nha a "li be ra lis mos desordenados”. Aspalavras disciplina e civilização foram evocadas para legitimar a forma de governo.

Esse Estado estaria acima dos interesses in divi du a is e de partidos po lí ti cos. Aso cie da de saudável seria aquela que observasse civismo, edu ca ção, dis ci pli na. Assim, vida pri va da e públi ca con fun diam-se no sen ti do da le gi ti ma ção des se po der de Esta do. Daí, sua in sis tên cia em normatizar a vida da família, abrangen do inúme ros es pa ços da exis tên cia in fan til.

0 olhar para as crianças pobres foi interpre ta do com base em ati tu des con si de ra das "beneméritas" dealguns ho mens de posses, "des providos de so ber ba” (VidaDoméstica, jan. 1952). A pobreza,em VidaDoméstica e Fon-Fon!, apareceu a partir da filantropia, sendo atendida por meio de obra ge ne ro sa de fi gu ras que ti nham certo destaque social, inclusive os governantes, e dos ricos, portadores de “compaixão" pelos ne cessita dos, cons ti tuin do uma ima gem dainfân cia como base da ri que za da na ção.

Valorizava-se a pobreza, na medida em quefosseconsidera dagenerosa eafe tiva. Nesse sentido, Vida Doméstica publicou um texto in ti tu la do "Uma De di ca ção Vale Mais do que a For tu na”, abor dan do a his tó ria de uma fa mí lia de pos ses, que co lo cou a mãe, já ido sa, em asilo sofisticado, deixando-a ao abandono: sua sa ú de fi cou ain da mais fra gi li za da devi do à au sên cia dos fi lhos. Ao mes mo tem po, apresentava uma experiência de mulher po bre, que vi via so zi nha, com mãe do en te, mere ce do ra de to dos os cui da dos da filha ze lo sa, capaz de assumir, apesar da ausência de re cursos financeiros, a do en ça ma terna. Resulta va des sa nar ra tiva a mo ral de que o di nhe i ro não era tudo, importando muito mais a dimen são afe tiva da re la ção fa mi li ar ( Vida Do méstica, out. 1942).

De acor do com o proje to ge ral de Vida Doméstica para a fa mília, os la ços mãe-filhos eram reforçados na relação inversa, quando os fi lhos de vi am cu i dar da mãe. lsso im pli cou uma ide a li za ção da po bre za mo ra li za da e feliz, que não dependia de grandes recursos eco nô mi cos para pra ti car o bem.

A rela ção mãe-filhos sem pre fun ci o nou como ape lo nas re por ta gens que tra ta vam da pobreza. Em Fon-Fon!, o tema foi também co men ta do a par tir da ex pe riên cia da Se gun da Gu er ra Mun di al. Para isso, fo ram pu blica das fotografias de crianças italianas pobres, apontando a habitação como um dos principais problemas resultantes da guerra: "Esses ga ro ti nhos des cal ços, sujos e es far ra pa dos de hoje são os cidadãos da ltália de amanhã" (Fon-Fon!, abr. 1949).

Essa vi são da in fân cia, re por tan do-se a um an ti-modelo da cri an ça hi gi e ni za da e com larbem es tru tu ra do, era uma pers pec tiva que tam bém se ti nha para o Bra sil, em bo ra aque les textos e imagens dessem a impressão de que tais problemas estivessem muito afastados do país. Pen sar na in fân cia, por tan to, era projetar o futuro desejável, que não se con fundia com aquele presente. 0 contexto da Segunda Guerra Mundial foi apresentado como pe ri go e ame a ça para a cri an ça, em seu 
nome deveriam ser evitadas ações belicosas (Fo lha da Ma nhã, 19 set. 1942).

\section{Conclusões}

As imagens fotográficas de crianças nas revistas Vida Doméstica e Fon-Fon! estavam sintonizadas com artigos e outros textos que va lo riza vam uma fa mília bem es tru tu ra da, com fi lhos sa u dá ve is. 0 que se en con tra va fora desses padrões era visto como desvio e devia ser corrigido.

Tais re vis tas da vam voz a mé di cos, edu ca do res e ou tros pro fis si o na is como ali a dos da imprensa no que se refere à preservação e trans for ma ção da re a li da de. Ini cia tivas que representavam construir possibilidades de afirma ção doside a is pro pos tos eram divul ga dase de fen di das pe los pe rió di cos, como ocor ria com par ques in fan tis e se ções de pu e ri cul tu ra, dentre outras.

A fa mília, para che gar aos pa drões ide a is ali defendidos, devia participar de um projeto de edu ca ção que se ini ci a va an tes do ca sa mento. Daí, a in sis tên cia nas re co men da ções so bre o par ce i ro ide al e es tra té gi as de pre serva ção do ca sa men to, critican do even tu a is desvi os em rela ção a es ses proje tos - fe mi nis mo, tra ba lho da mu lher fora do lar etc. Ape sar dis so, as re vis tas lidavam com conflitos socialmente presentes, anun cian do o tra ba lho fe mi ni no em pro pa gandas, e as su min do ativi da des as sis ten ci a is como tarefas femininas que se desdobravam para fora do lar tam bém no cu i da do com as cri an ças. Ao mes mo tem po queva lo riza vam trajesluxu osos e vida so ci al ativa, con de na vam a ex ces si va de di ca ção a es sas ativi da des quan do re pre senta vam prejuízo ao bom convívio fa mi liar.

Po de-se iden ti fi car que, do pon to de vista das revis tas aqui dis cu ti das, a har mo nia no lar era dada pe los cu i da dos e ori en ta ções ci entífi cas a de qua das; fora dele, isso foi abor da do a partir da escolaridade, que começa va nos materna is, passan do pelos parques in fan tise atingin do o en si no fun da men tal.
Vida Doméstica referiu-se à Conferência Na ci o nal de Pro te ção à Infân cia de 1933, publicando trecho que comentava a fala de Lucilia Ribeiro:

O lar deve ser para a creança, uma fonte de ale gria; ali deve re ce ber o pri me i ro to que para a formação do seu caracter. Entre carinhos, mas tam bém jus tas e se ve ras re pre hen sões, é que a ver da de i ra mãe, se gun do Chris to, for ma os tenros corações de seus filhinhos; se guia-lhes os pas sos ain da va cil lan tes e in certos, guia-lhes tam bém a alma 'the sou ro di vino' que Deus, Suprema auctoridade, de po si tou-lhe nas mãos. Os paes de vem ser os cons tan tes orien ta do res de seus filhos; devem fazer da creança, por uma instrucção sabia e cri te ri o sa, um ente ca paz de apre ciar a Vir tu de, a fim de nos seus ac tos, pra ti ca-los. (Vida Doméstica, fev. 1936)

Esse trecho é muito representativo em re la ção às ima gens de in fân cia ve i cu la das pelos periódicoscomentados. Ele con tém a expressão "verdadeira mãe", qualificando a quem se re fe ria, si tu an do-a em re la ção a Cristo. Atribui aos pais uma permanente função ori en ta do ra, em nome de Deus, e co lo ca como fru to da cor re ta ori en ta ção a prá ti ca da vir tude, devidamente sacralizada.

Em relação à infância, os periódicos de sen volve ram uma cul tu ra fo to grá fica, que atuava em mo men tosso cialmen te privilegiados - prime ira Co mu nhão, fes tas de en cer ra mento do ano letivo, carnaval - e também num co ti di a no que a fir ma va a be le za e o su cesso. Atra vés das fo to gra fi as, cons ti tuía-se um es pe tá cu lo so ci al do que se de via ver.

Ao mesmo tempo, havia cortiços, abandonos, trabalho infantil: essas outras faces da criança idealizada, higienizada e bela, que, em bo ra ten do a exis tên cia ad mi tida, pou co apa re ce ram em VidaDoméstica e Fon-Fon! 


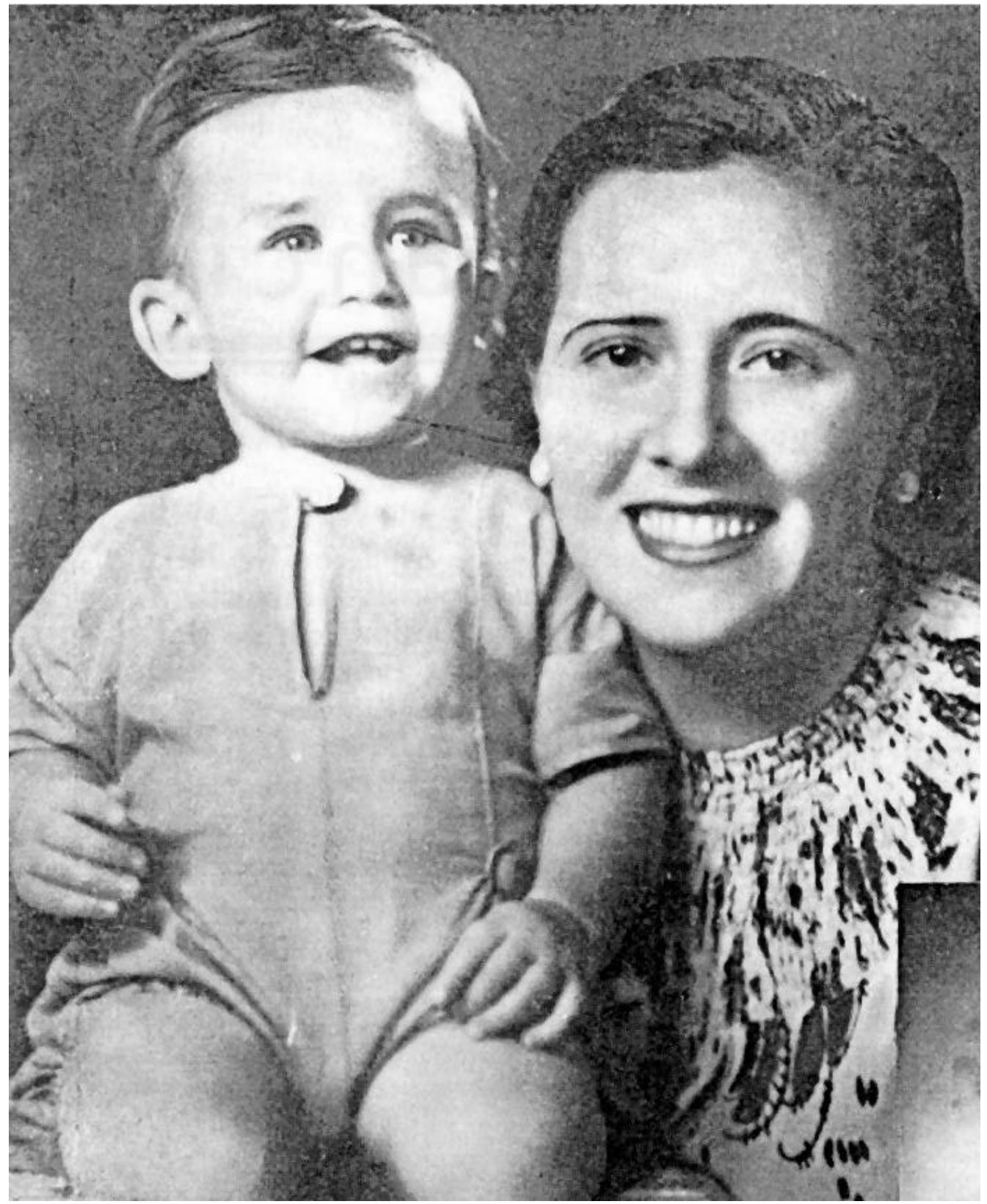

Infância, out. 1936

\section{Referências bibliográficas}

BAHIA, Juarez. Históriadaimprensabrasileira. São Pa u lo: Áti ca, 1990. 2 v.

BARTHES, Ro land. Acâma racla ra. Rio de Janeiro: Nova Fron te i ra, 1996.

BASSANEZI, Car la. Reven do as mu lhe res: re vis tas fe mi ni nas e re la ções ho mem-mulher (1945/1964). Rio de Janeiro: CivilizaçãoBrasileira, 1996. 
CAPELATTO, Ma ria He le na. Pro pa gan da po líti ca e cons tru ção da iden ti da de na ci o nal coletiva. Revista Brasileira de História, São Pa u lo, v.31/32, n.328/352, 1996.

DIAS, Ma ria Odi la Sil va. Quo tidia no e po derem São Pa ulo no sécu lo XIX. São Pau lo: Bra sili en se, 1984.

FOLHA DA MANHÃ. São Pau lo.

FON-FON! . Rio de Janeiro.

KOSSOY, Bo ris. Fotografiaehistória. São Pa u lo: Áti ca, 1989.

MACHADO, Arlin do. Ailu sãoespecular .SãoPau lo: Bra silien se, 1984.

PAUL0, He loísa He le na de Je sus. ODIP eajuven tu de: ide o lo gia e pro pa gan da es ta tal (1939/1945). RevistaBrasileirade História, São Pa u lo, v.14, n.99/113, mar/ago. 1987.

SANT'ANNA, Denise Bernuzzide. Propagan da ehis tó ria: antigos proble mas, novas quest ões. ProjetoHistória, São Pau lo, v.14, n.89/112, 1997.

SANTOS, Carlos José Ferreira dos. Nem tudo era italiano: São Paulo e pobreza (1890/1915). São Paulo: Anna Blu me/FAPESP, 1989.

SCHEMMES, Cláudia. Festascívicaseesportivasnopopulismo:umestudocomparativodosgovernos Vargas (1937/1945) e Pe rón (1946/1955). SãoPaulo, 1995. Dis sertação (Mestra do) Facul da de de Filo sofia, Letras e Ciências Humanas da USP.

SILVA, Mar cos A. da. Caricatarepública:Zé povo e o Bra sil. São Pau lo: MarcoZero/CNPq, 1990. (Onde Estáa Re pú bli ca?)

SODRÉ, Nel son Wer neck. His tó ria da im pren sa no Bra sil . 2. ed. Rio de Janeiro: Gra al, 1977.

VIDADOMÉSTICA. Rio de Janeiro.

Recebido em 31.08 .00

Apro va do em 07.11 .00

Olga Bri tesé pro fes so ra do pro gra ma de Pós-Graduação em His tó ria da Pon ti fícia Uni ver si da de Cató li ca de São Pau lo, nas áre as de His tó ria do Bra sil e Pes qui sa His tó rica. Dou to rou-se em His tó ria so cial na mesmauniver sidade, soborientação da Pro fa. Dra. Déa R. Fe ne lón. 